\title{
Carbohydrate Antigen 19-9-Positive Gastric Adenocarcinoma: Autopsy Findings and Review of the Literature
}

\author{
Tan Wang ${ }^{a, b}$ Yoko Matsuda ${ }^{b}$ Atsuko Seki ${ }^{b}$ Keisuke Nonaka ${ }^{b}$ \\ Mototsune Kakizaki ${ }^{b} \quad$ Daita Kaneda $^{c}$ Junko Takahashi-Fujigasaki ${ }^{c}$ \\ Shigeo Murayamac Tomio Arai ${ }^{\mathrm{b}}$ \\ ${ }^{a}$ Department of Comprehensive Pathology, Graduate School of Medical and Dental \\ Sciences, Tokyo Medical and Dental University, Tokyo, Japan; ${ }^{b}$ Department of Pathology, \\ Tokyo Metropolitan Geriatric Hospital, Tokyo, Japan; 'Department of Neuropathology, \\ Tokyo Metropolitan Institute of Gerontology, Tokyo, Japan
}

\section{Keywords}

Gastric Cancer · Carbohydrate antigen19-9 · Autopsy · Biomarker

\begin{abstract}
Carbohydrate antigen 19-9 (CA19-9) is a well-known tumor marker for pancreatobiliary cancer, and several studies have shown that an elevated serum CA19-9 level is associated with more aggressive biological behavior in gastric cancer (GC). However, the clinicopathological characteristics of CA19-9-positive GC remain unclear. We herein report an autopsy case of CA19-9-positive GC in an 84-year-old man who was admitted to our hospital because of paralysis and anemia. Autopsy revealed an ulcerative-invasive tumor measuring $72 \times 60 \mathrm{~mm}$ in the anterior wall of the gastric body. The tumor had invaded beyond the muscularis propria, and metastasized to the lung, liver, and regional lymph nodes. Histologically, the tumor cell had oval nuclei with abundant clear cytoplasm, and tubular and/or papillary features with
\end{abstract}




\section{Case Reports in \\ Gastroenterology}

Case Rep Gastroenterol 2017;11:545-553

(c) 2017 The Author(s). Published by S. Karger AG, Basel www.karger.com/crg

Wang et al: Carbohydrate Antigen 19-9-Positive Gastric Adenocarcinoma: Autopsy

Findings and Review of the Literature

prominent lymphovascular permeation and perineural invasion, mimicking pancreatobiliary carcinoma. Immunohistochemically, the tumor cells showed diffuse immunopositivity for CA19-9 and carcinoembryonic antigen. According to a review of cases reported in the literature, CA19-9-positive GCs show clinicopathological characteristics such as antral location, ulcerative-infiltrating gross feature, differentiated histology, prominent lymphatic and venous invasion, higher proportion of metastasis, and higher clinical stage. These results suggest that CA19-9-positive GC is pathologically a distinctive type of tumor with aggressive biological behavior.

(C) 2017 The Author(s)

Published by S. Karger AG, Basel

\section{Introduction}

Carbohydrate antigen 19-9 (CA19-9), also known as sialyl Lewis antigen, is a wellknown tumor marker for the screening and identification of pancreatobiliary cancer and for the detection of carcinomas of the digestive tract. Among digestive tract cancers, 7.3-18\% of gastric cancer (GC) cases show high CA19-9 levels in serum [1, 2], and several studies have shown an association between an elevated serum CA19-9 level and aggressive biological behavior of GC [1-3]. In addition, an immunohistochemical study revealed that CA19-9 has a 17.3\% immunopositivity rate in GC specimens [4]. However, the clinicopathological characteristics of CA19-9-positive GC remain unclear. We herein report an autopsy case of CA19-9positive GC in an elderly male patient. We aimed to clarify the clinicopathological characteristics of the tumor, such as tumor distribution, metastatic pattern, and other complications. Because most previous reports dealt with surgically resected specimens, metastatic lesions and/or serious complications were not examined pathologically. However, since we performed an autopsy in the present case, which included an examination of the whole body, we were able to investigate these factors. We also reviewed reported cases of CA19-9-positive GC and compared them with conventional GCs.

\section{Case Report}

An 84-year-old man with a noncontributory medical history was admitted to our hospital because of bilateral paralysis of the legs and dysarthria. No apparent abnormalities were found on physical examination at admission, except for muscle weakness in the legs. Hematological tests showed severe anemia (hemoglobin, $7.0 \mathrm{~g} / \mathrm{dL}$ ) and an elevated white blood cell count $\left(9.35 \times 10^{3} / \mu \mathrm{L}\right)$. Laboratory tests showed shortened activated partial thromboplastin time $(24.0 \mathrm{~s})$, mild renal dysfunction (urea nitrogen, $29 \mathrm{mg} / \mathrm{dL}$; creatinine, 1.42 $\mathrm{mg} / \mathrm{dL})$, and elevated levels of serum lactate dehydrogenase (298 IU/L), $\gamma$ glutamyltransferase (104 IU/L), carcinoembryonic antigen (CEA; $401.0 \mathrm{ng} / \mathrm{mL}$; normal level $<6.0 \mathrm{ng} / \mathrm{mL}$ ), and CA19-9 (171 U/mL; normal level <37 U/mL). Magnetic resonance imaging and computerized tomography scans showed scattered small cerebral infarcts (Fig. 1a) and a large mass in the stomach with regional lymph node and liver metastases (Fig. 1b). Gastric endoscopy revealed an ulcerative-invasive tumor in the anterior wall of the gastric body, which was identified as a tubular adenocarcinoma on biopsy. The case was clinically diag- 


\section{Case Reports in \\ Gastroenterology}

Case Rep Gastroenterol 2017;11:545-553

(c) 2017 The Author(s). Published by S. Karger AG, Basel www.karger.com/crg

Wang et al: Carbohydrate Antigen 19-9-Positive Gastric Adenocarcinoma: Autopsy Findings and Review of the Literature

nosed as advanced GC (stage IV) and Trousseau syndrome. We administered the best supportive care for the cerebral infarction and malnutrition. The patient developed right hemiplegia 3 months after admission, and then died of the tumor. Autopsy was performed after obtaining permission from the family member of the patient.

Autopsy revealed an ulcerative-invasive tumor measuring $72 \times 60 \mathrm{~mm}$ in the anterior wall of the gastric body. The tumor invaded beyond the muscularis propria with expansive growth, but the invasive front directly involved the pancreas and metastasized to the regional lymph nodes, lungs, and liver. Histologically, the tumor showed a papillary and/or tubular structure, and the constituent tumor cells had oval nuclei with abundant clear cytoplasm with mucin (Fig. 2), mimicking the morphology of pancreatobiliary carcinoma. The tumor showed prominent lymphatic and venous permeation, and occasionally invaded along the peripheral nerve in the subserosal layer, which was estimated to be perineural invasion. Although the tumor metastasized to regional lymph nodes, lungs, and liver, no primary cancers were found in the pancreas or the biliary tract. Immunohistochemically, the tumor cells demonstrated diffuse immunopositivity for CA19-9 (Fig. 3a) and CEA (Fig. 3b). Regarding the expression of mucin core proteins, the tumor cells were diffusely positive for MUC1 at the luminal surface (Fig. 3c) with occasional cytoplasmic reaction (Fig. 3d), especially in poorly differentiated areas, and were focally positive for MUC5AC and negative for MUC2 (Fig. 3e) and MUC6 (Fig. 3f). Nonbacterial thrombotic endocarditis involved the mitral valve. The lesion induced numerous small infarcts with fibrin thrombi with occasional organization in the brain.

\section{Discussion}

In this report, we described the pathological features of an autopsy case of CA19-9positive GC. The histological findings and biological behavior of the tumor were similar to those of pancreatobiliary carcinoma. Thus, CA19-9-positive GC shows distinctive clinicopathological characteristics and complications in comparison with those of conventional GCs.

CA19-9 is generally produced by epithelial cells of the pancreas, biliary tract, or gallbladder, and is rarely secreted by normal gastric mucosa [5]. Most of the CA19-9 secreted by these cells is metabolized in serum, resulting in very low serum concentration in healthy individuals. However, in cases of pancreatobiliary carcinoma, epithelial cells of the tumor can produce a large amount of CA19-9, resulting in an increased serum CA19-9 level. In the present case, we found strong and diffuse immunopositivity for CA19-9 in the cytoplasm of GC cells, suggesting that the gastric tumor could produce a large amount of CA19-9.

Previous studies found that elevated serum CA19-9 was observed in a relatively higher proportion (7.3-18\%) of patients with GC [1, 2]. Numabe [4] investigated CA19-9-positive GCs biochemically and immunohistologically, and reported that the number of GC cases with elevated serum CA19-9 levels was 1.4-fold the number of cases with CA19-9 immunopositivity. The reason for this discrepancy is currently unclear, and warrants further examination.

The present case demonstrated prominent lymphatic and venous invasion and hematogenous metastases to the lung and liver. As CA19-9-producing cancer cells could preferentially bind to selectin on endothelial cells, these cancer cells could potentially metastasize 


\section{Case Reports in \\ Gastroenterology}

Case Rep Gastroenterol 2017;11:545-553

(c) 2017 The Author(s). Published by S. Karger AG, Basel www.karger.com/crg

Wang et al.: Carbohydrate Antigen 19-9-Positive Gastric Adenocarcinoma: Autopsy Findings and Review of the Literature

hematogenously. This phenomenon could also induce dysfunction of the coagulation system, resulting in nonbacterial thrombotic endocarditis and/or Trousseau syndrome, as seen in the present case. Because of this tendency, CA19-9 could have significant prognostic value in $\mathrm{GC}[3,6,7]$.

The histopathological features of pancreatobiliary carcinoma generally differ from those of GC. Constituent cells of pancreatobiliary carcinoma commonly have clear cytoplasm with mucin. However, in the present case, the results of histology and the features of the clinical course, such as hypercoagulation status and Trousseau syndrome, were similar to those of pancreatic cancer and its complications. Thus, this type of tumor might share both histopathological features and a similar clinical course with pancreatic cancer.

GCs can be classified into several phenotypes such as gastric, intestinal, and mixed type according to the expression of mucin core proteins. In the present GC case, we detected prominent MUC1 expression with occasional MUC5AC expression and no expression of MUC2 or MUC6. This expression pattern is similar to that seen in pancreatobiliary carcinoma. Consequently, the tumor demonstrated a similar mucin phenotype to pancreatobiliary carcinoma, as well as similar morphology.

To provide an overview of the clinicopathological features of CA19-9-positive GC, we compiled the data from 65 previously reported cases. We retrieved these cases using PubMed and Ichushi (or Japan Medical Abstract Society) Web by performing a search using the keywords "gastric cancer" and "CA19-9" to identify papers published between 1985 and 2016. Although the reports included papers written in both English and Japanese, most of the patients with CA19-9-positive GC were Japanese because most of these cases were reported by Japanese investigators [8-10]. We excluded metastatic CA19-9-positive GC cases. Among these cases, including our case, the mean patient age was 68.3 years (range, 26-85 years) with male predominance ( 44 males and 22 females; male to female ratio 2:1). A subanalysis of these cases revealed that CA19-9-positive GCs preferentially occurred in the lower third of the stomach in 34 cases (57.6\%). Tumor size ranged from 12 to $150 \mathrm{~mm}(<50 \mathrm{~mm}$, 16 cases; $\geq 50 \mathrm{~mm}, 33$ cases). Compared with conventional GCs [11], CA19-9-positive GCs demonstrated distinct clinicopathological characteristics such as antral location, differentiated histology, prominent lymphatic and venous invasion, higher proportion of lymph node metastasis, and advanced stage (Table 1). Thus, these data suggest that CA19-9-positive GC is a distinct tumor with aggressive biological behavior. However, a few reports demonstrated specific features of CA19-9-producing GCs such as GC arising in hyperplastic foveolar hyperplasia [12,13] and carcinoma of the remnant stomach [14].

In conclusion, CA19-9-positive GC is a distinctive type of tumor, with clinicopathological features that differ from those of conventional GCs. In addition, its biological behavior, such as prominent perineural invasion, lymphatic permeation, and the occurrence of Trousseau syndrome as a complication, are similar to those of pancreatobiliary cancer. Further studies are needed to clarify the clinicopathological characteristics of CA19-9-positive GC. 


\section{Acknowledgments}

We thank the staff of the Department of Pathology for their technical assistance. This study was supported in part by the JSPS KAKENHI (grant No. JP25460428 and JP16K08664 to T.A.).

\section{Statement of Ethics}

There are no ethical conflicts to declare. Informed written consent was obtained from the family member of the deceased person.

\section{Disclosure Statement}

The authors have no competing interests to declare.

\section{References}

1 Wada N, Kurokawa Y, Miyazaki Y, Makino T, Takahashi T, et al: The characteristics of the serum carcinoembryonic antigen and carbohydrate antigen 19-9 levels in gastric cancer cases. Surg Today 2017;47:227-232.

-2 Ishigami S, Natsugoe S, Hokita S, Che X, Tokuda K, et al: Clinical importance of preoperative carcinoembryonic antigen and carbohydrate antigen 19-9 levels in gastric cancer. J Clin Gastroenterol 2001;32:41-44.

-3 Song YX, Huang XZ, Gao P, Sun JX, Chen XW, et al: Clinicopathologic and prognostic value of serum carbohydrate antigen 19-9 in gastric cancer: a meta-analysis. Dis Markers 2015;2015:549843.

4 Numabe K: Serum and immunohistological examination of CA19-9 positive gastric cancer (in Japanese with English abstract). Showa Univ J Med Sci 1993;53:146-155.

5 Lin F, Prichard J: Handbook of Practical Immunohistochemistry: Frequently Asked Questions. New York, Springer, 2011.

-6 Kwon OK, Yu W, Chung H: Prognostic value of postoperative CA19-9 normalization in patients with advanced gastric cancer. Hepatogastroenterology 2013;60:240-243.

-7 Dilege E, Mihmanli M, Demir U, Ozer K, Bostanci O, et al: Prognostic value of preoperative CEA and CA 19-9 levels in resectable gastric cancer. Hepatogastroenterology 2010;57:674-677.

8 Kato K, Taniguchi M, Kawakami T, Nagase A, Matsuda M, et al: Gastric cancer with a very high serum CA19-9 level. Case Rep Gastroenterol 2011;5:258-261.

9 Mizuguchi K, Sato K, Maekawa H, Sakurada M, Orita H, et al: A case report of carbohydrate antigen 19-9 producing advanced gastric cancer. Cancer Clin Oncol 2016;5:49-53.

10 Iwase T, Imai Y, Onishi K, Kato K, Kanemasa, et al: A case of carbohydrate antigen 19-9 producing gastric mucinous adenocarcinoma with the appearance of a submucosal tumor (in Japanese with English abstract). Nippon Shokakibyo Gakkai Zasshi 1997;94:746-750.

11 Japan Gastric Cancer Association: Report of nation-wide analysis of gastric cancers registered in Japan, 2008. http://www.jgca.jp/entry/iganhtml/doc/2008_report.pdf.

12 Guo X, Yamada S, Omori H, Wang K-Y, Tasaki T, et al: CA19-9 producing early gastric adenocarcinoma arising in hyperplastic foveolar poly a very unique resection case. Diagn Pathol 2011;6:112.

13 Nagai K, Ishii K, Matsumaru K, Kohra T, Okajima T, et al: Malignant transformation of Nakamura type IV gastric polyp with CA 19-9 production. J Gastroenterol 1998;33:91-96.

14 Tahara H, Hayasi S, Kusano M, Kojima M, Ohki I: A case of CA19-9-producing adenocarcinoma of the remnant stomach resembling gastritis cystica polyposa. Dig Endosc 2005;17:175-178. 


\section{Case Reports in Gastroenterology}
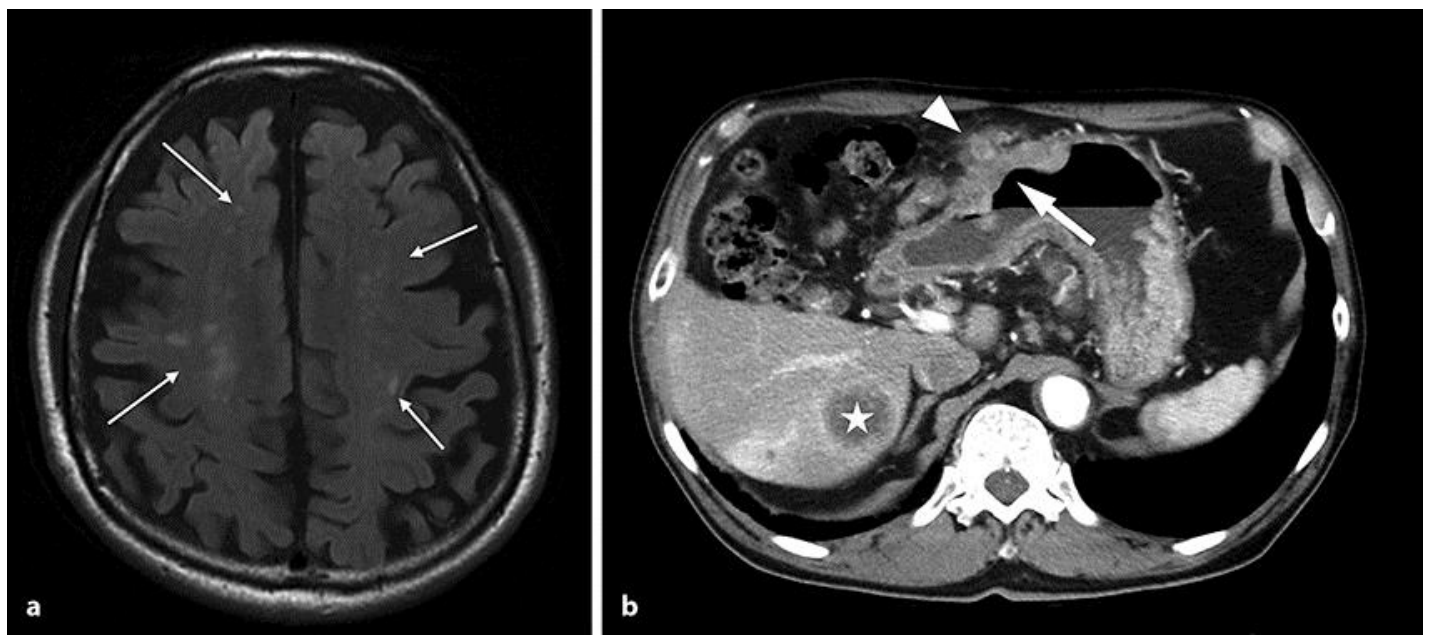

Fig. 1. Magnetic resonance imaging and computerized tomography scans. a Small cerebral infarcts (arrows) are scattered in the brain. b A large tumor (arrow) exists in the gastric wall with regional lymph node (arrowhead) and liver (asterisk) metastases. 


\section{Case Reports in Gastroenterology}

Case Rep Gastroenterol 2017;11:545-553
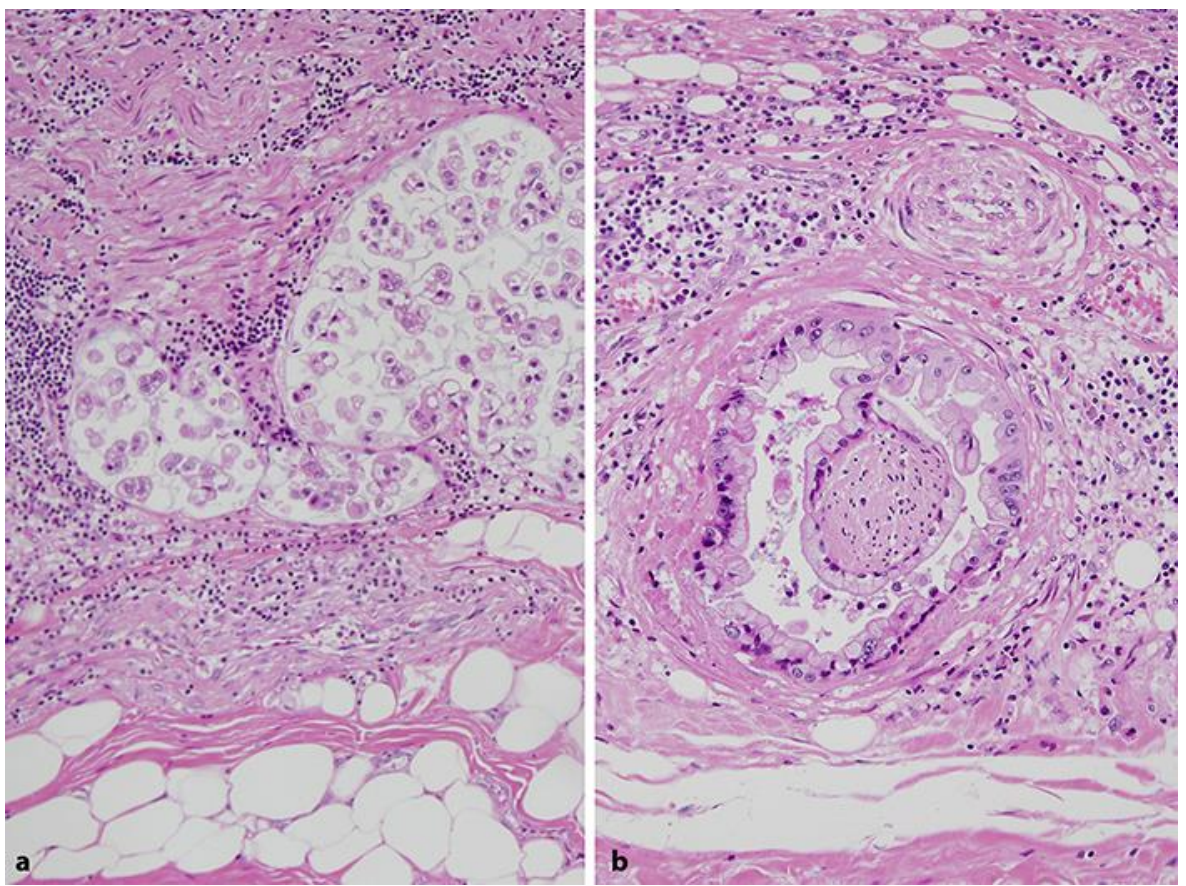

Fig. 2. Histopathological finding of CA19-9-positive gastric cancer. Note the papillotubular adenocarcinoma cells with clear cytoplasm, mimicking pancreatobiliary cancer. Hematoxylin-eosin staining. Original magnification, $\times 200$. 

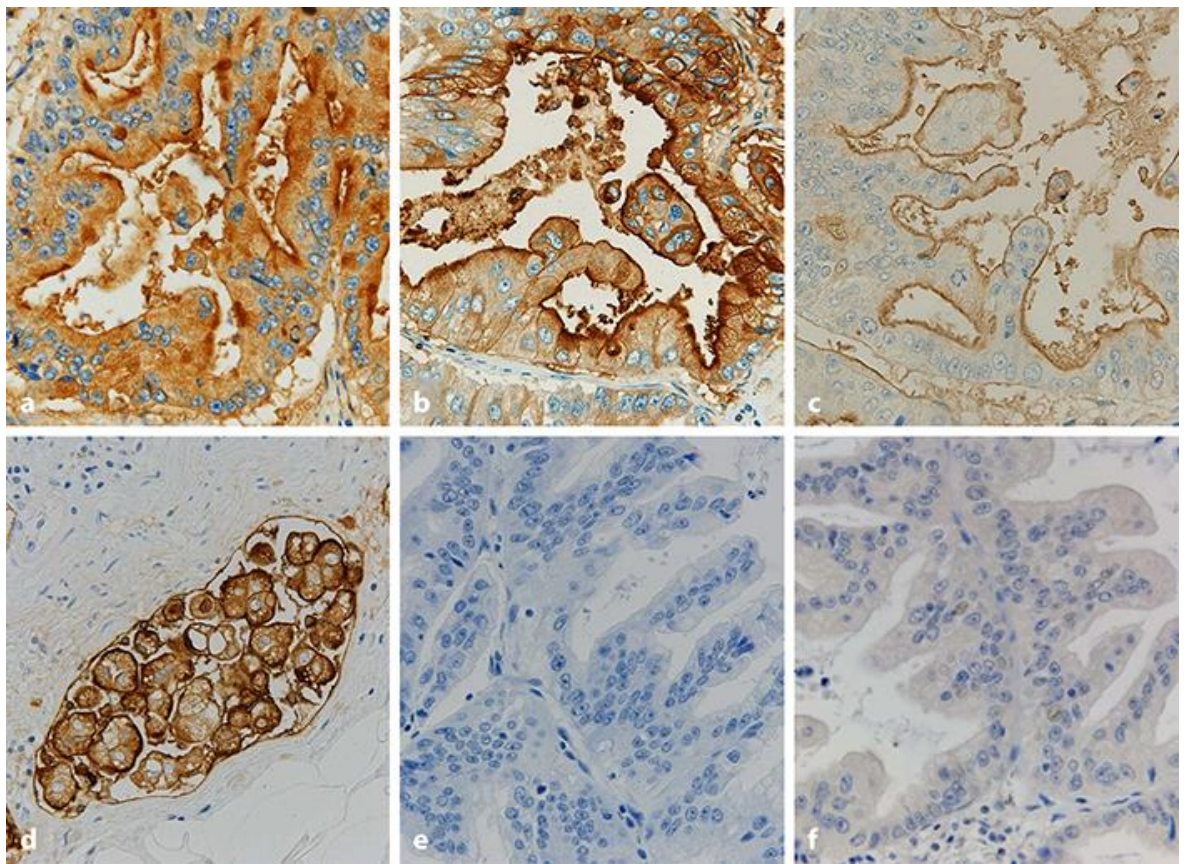

Fig. 3. Immunohistochemical findings of gastric cancer. The tumor shows diffuse immunopositivity for CA19-9 (a) and CEA (b). The tumor also shows immunopositivity for MUC1 at the luminal surface (c) and occasional immunopositivity in the cytoplasm (d) in the poorly differentiated component in lymphatic vessels. The tumor demonstrates negative immunohistochemical reactions of MUC2 (e) and MUC6 (f). Counterstaining, hematoxylin. Original magnification, $\times 400$. 
Table 1. Clinicopathological characteristics of CA19-9-positive gastric cancers

\section{Age, years}

Sex (male/female)

Location (U/M/L/diffuse)

Gross feature $(0 / 1 / 2 / 3 / 4 / 5)$

Histology (pap/tub/por/sig/muc)

Size $(<5 \mathrm{~cm} / \geq 5 \mathrm{~cm})$

T classification $(1 / 2 / 3 / 4)$

lymphatic invasion $(-/+)$

venous invasion $(-/+)$

Lymph node metastasis $(-/+)$
68.3 (26-85)

$44 / 22$

$7 / 8 / 34 / 2$

$12 / 9 / 12 / 19 / 5 / 7$

$6 / 31 / 13 / 6 / 6$

$16 / 33$

$16 / 3 / 11 / 24$

$7 / 37$

$13 / 29$

$8 / 29$

Data are presented as mean (range) or $n$, as appropriate. The data are summarized from 66 cases of CA19-9-positive gastric cancer, including 65 previously reported cases and the present case. Location, gross feature, histology, and $\mathrm{T}$ classification were according to the Japanese classification of gastric carcinoma [15]. U, upper third, M, middle third; L, lower third; pap, papillary adenocarcinoma; tub, tubular adenocarcinoma; por, poorly differentiated adenocarcinoma; sig, signet-ring cell carcinoma; muc, mucinous carcinoma. 